\title{
Study photometric properties of spiral galaxy NGC 4448 using CCD camera with multiband filters
}

\author{
Sinan H. Ali \\ Department of Astronomy and Space, College of Science, University of Baghdad \\ E-mail: sinan1914@gmail.com
}

\begin{abstract}
The goal of our study is to perform detailed multiband surface photometry of the spiral galaxy NGC 4448 and its brightest starforming regions. The structure and composition of the stellar population in the surface brightness galaxy NGC 4448 was studied using BVR CCD photometry. The observations were obtained on the $1.88 \mathrm{~m}$ optical telescope of Kottamia Astronomical Observatory (KAO), Egypt. A two-dimensional decomposition of the galaxy bulge and disk components is carried out. A powerful star forming region is observed near the galactic center. Based on the positions of the various components of the galaxy in two color diagrams. From the observations, the surface brightness profiles, Ellipticity profiles, position angle profiles and color indices profiles are described and studied.
\end{abstract} Key words

Spiral galaxy, surface photometry, ellipticity, color index.

Article info.

Received: Mar. 2017

Accepted: May. 2017

Published: Dec. 2017

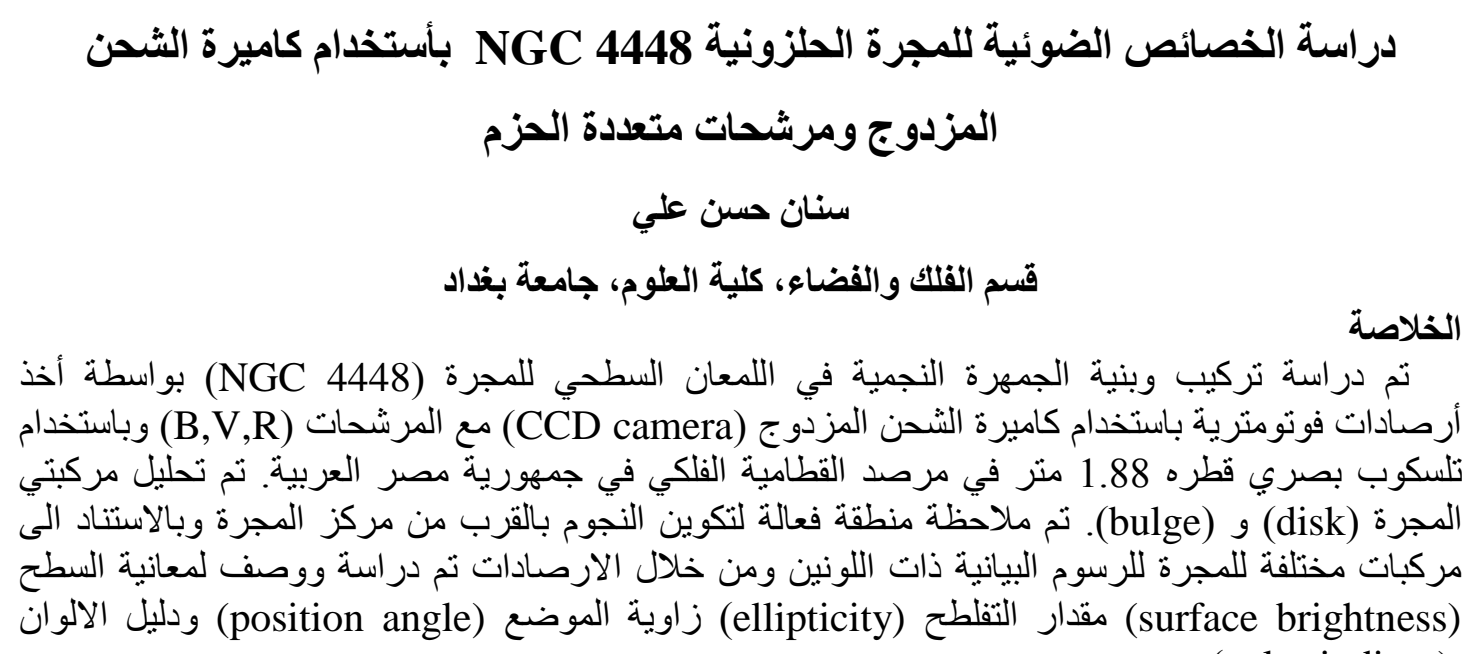

\section{Introduction}

The surface photometry of galaxies provides the fundamental and essential information for the study of their structures and dynamics. The recent advances in CCD device technology and image processing techniques allow us to deal the photometric data of faint objects of great deal with high precision. In this study, we performed the multiband surface photometry of galaxies with a CCD camera equipped on $1.88 \mathrm{~m}$ optical telescope of Kottamia Astronomical Observatory (KAO) Egypt.

The specific characteristics of their photometric structures will provide the essential information not only of the relations of stellar kinematics to configurations but also of the origin of the bar structure. Surface photometry is an important tool in the understanding of galaxy mass and structure. Obtaining the structural 
characteristics and the total luminosity of a galaxy requires knowledge of its surface brightness profile to a significant depth. During a surface photometry project to explore the structure of galaxies by morphological type (Schombert \& Smith 2011) [1].

Most of the current knowledge about the structure of galaxies came from the analysis of the photographic plates. Recently, this technique has been enhanced by the replacement of digital imaging. This new technology improves sensitivity by a factor of 100 and provides greater dynamic range. The 1980s saw the rise of the CCD cameras, it is a device consists of light sensitive photoelectric cells, or "pixels", that transfer incoming light particles into electric current [2]. The pixels can also be digitally combined, which increases light sensitivity at the expense of resolution [3]. The light observed in the visual wavelengths from a galaxy arises from all its stars, with the radiation contribution from the brightest hot stars competing with the light from the more numerous fainter cool stars. In this sense, we can infer a galaxy's stellar composition and age by observing its color [4]. Photometry can be defined as the determination of the amount and temporal nature of the flux emitted by an astronomical object as a function of wavelength [5]. By taking images of a galaxy at different wavelengths, and then analyzing the intensity distribution in each image, one can determine the distribution of stars, dust, and gas within the object. In order to obtain images of objects at different wavelengths for photometric study, a series of filters are introduced into the light path of the instrument being used. In order to be scientifically useful for the resultant images, it is important that a standard set of filters be used. One such standard is the Cousins set. The set contains five filters UBVRI filters. It is not necessary to use all five filters in order to gather photometric data [6]. In this paper we use only three filters (BVR). Surface photometry is an important tool in understanding the galaxy mass and structure. Obtaining the structural characteristics and the total luminosity of a galaxy requires knowledge of a surface brightness profile. During a surface photometry project to explore the structure of galaxies by morphological type [1].

\section{Observations, data reduction and photometric calibration}

In this section we will concentrate on the kind of optical telescope, the processing that implied to manipulate the data and photometric calibration.

\section{Observations}

The observations were obtained in March 2011at the Newtonian focus of the $(1.88 \mathrm{~m})$ reflector telescope which has a focal length of $(9.15 \mathrm{~m})$ at KAO Egypt. With broadband B, V, and R this CCD array realizes a photometric system close to the standard JohnsonCousins UBVRI system. The array was cooled with liquid nitrogen. The size of the array is $1.024 \times 1.024$ pixels, providing an image scale of 0.305 arcsec/pixel. Table 1 illustrates an observing log. 
Table 1: Log of observations.

\begin{tabular}{|c|c|c|c|c|c|}
\hline $\begin{array}{c}\text { Date of } \\
\text { observation }\end{array}$ & $\begin{array}{c}\text { The name of } \\
\text { the Galaxy }\end{array}$ & Filters & $\begin{array}{c}\text { Time exposure } \\
(\mathrm{sec})\end{array}$ & Seeing & air mass \\
\hline $2011 / 3 / 25-26$ & NGC 4448 & $\mathrm{~B}$ & $5 \times 600$ & $1.81 "$ & 1.33 \\
& & $\mathrm{~V}$ & $5 \times 600$ & & 1.36 \\
& & $\mathrm{R}$ & $5 \times 600$ & & 1.41 \\
\hline
\end{tabular}

\section{Data reduction}

We performed the preliminary reduction of the images. To correct for electron bias and the effect of "hot pixels" and bad columns of the CCD array, a dark frame that was the average of several exposures taken with a closed shutter and the same integration time as for the object subtracted. Summed frames of the twilight sky are used, then divided each image by its flat field to correct for the nonuniform sensitivity of the detector pixels. To performed the subsequent data reduction by using a standard procedure incorporating

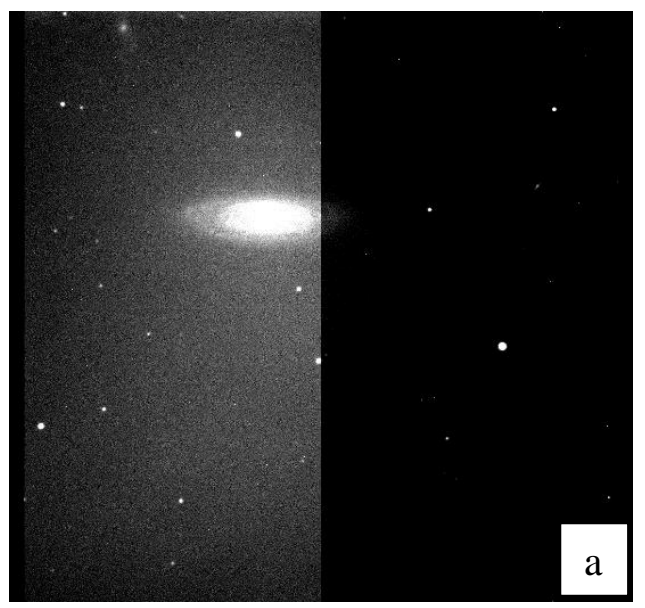

Astronomical Image Processing Packages software IRAF [7-11].

\section{Photometric calibration}

The photometric calibration of the galaxies NGC 4448 is based on standard stars PG 1633+099; from the list of the Landolt standard stars [12] observed on the each night, the instrumental magnitudes were transformed to the standard JohnsonCousins UBVRI system. The images of galaxies NGC 4448 is recorded on CCD camera using $\mathrm{B}, \mathrm{V}$ and $\mathrm{R}$ filters. These images are demonstrated in Fig.1.

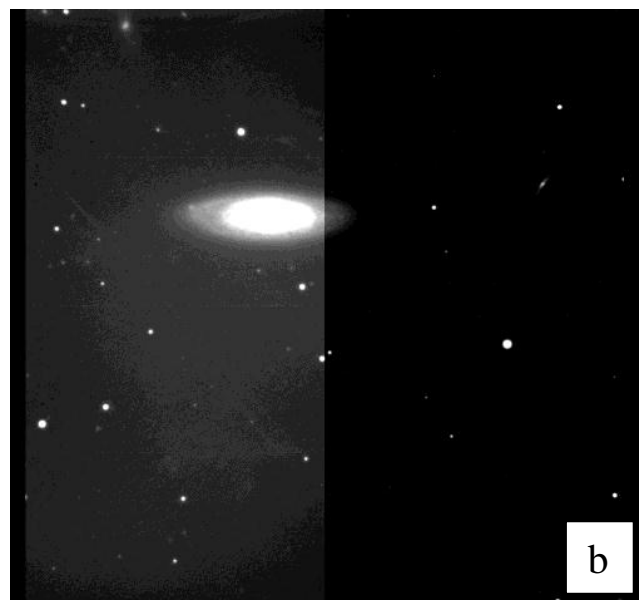

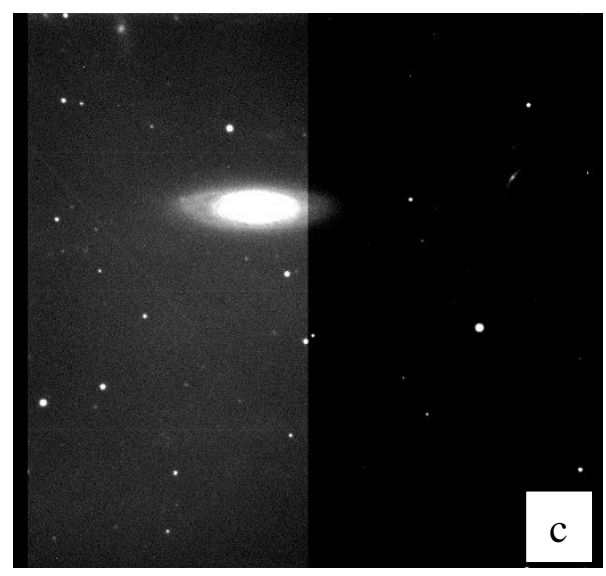

Fig.1: CCD image of NGC 4448 before reduction (a) B filter (b) V filter (c) R filter. 
The output of the data reduction process for the galaxies NGC 4448 are

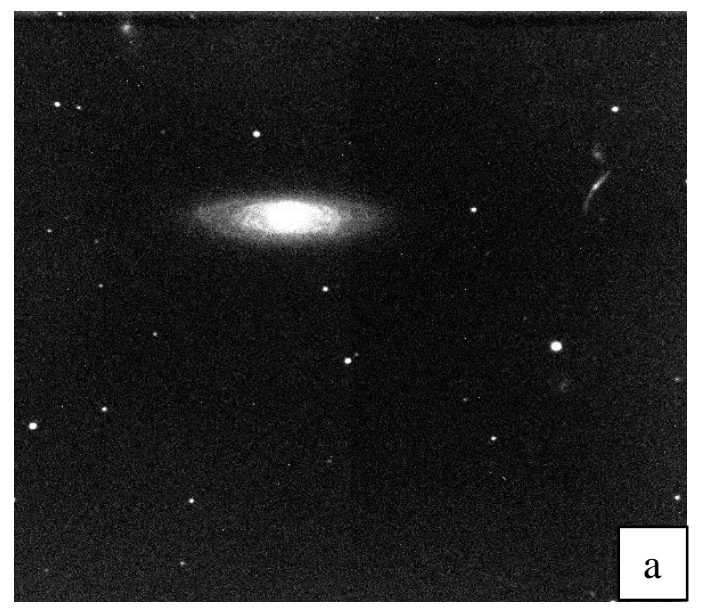

shown in Fig. 2. The results illustrate a clean background

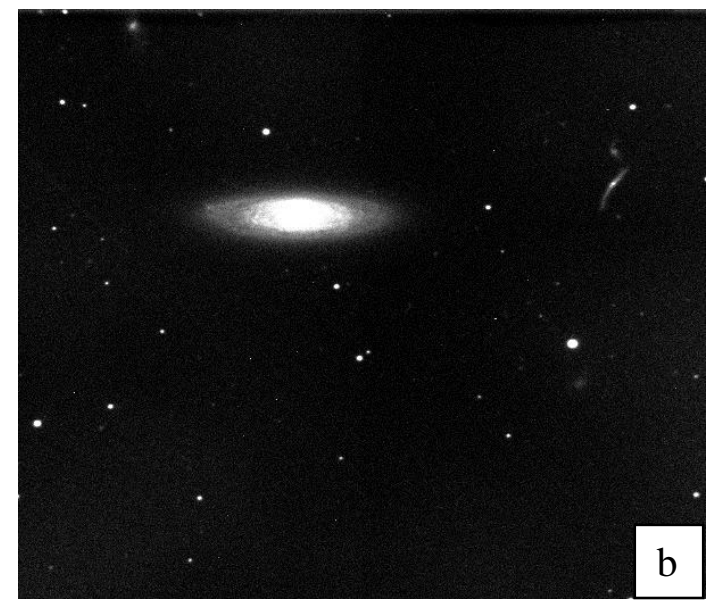

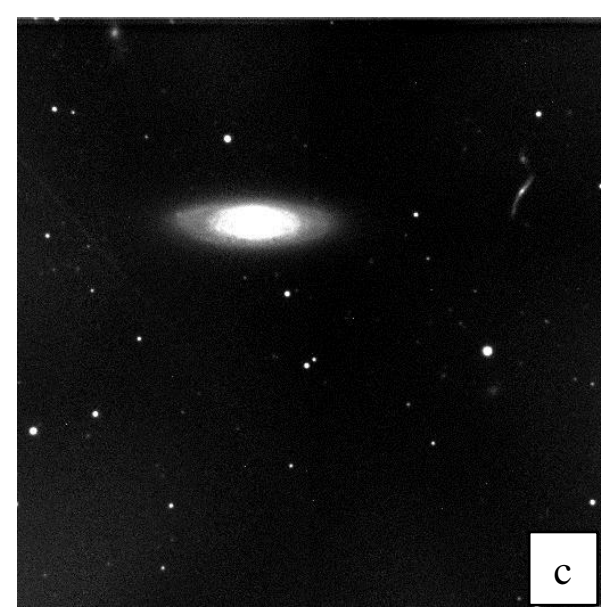

Fig. 2: CCD image of NGC 4448 after reduction (a) B filter (b) V filter (c) R filter.

\section{Data analysis and results}

NGC 4448 is a barred spiral galaxy with a prominent inner ring structure in the constellation Coma Berenices. Fig.3 represents the photometric profiles along the major axis of the galaxy NGC 4448 using B, $\mathrm{V}$ and $\mathrm{R}$ filters which are symmetric about the center. It's so clear that the galaxy possesses a large bright core. From this figure the diameter of the nucleus and the bulge are estimated and they were found to be 8 and 14 arcsec respectively. A way from the center of the bulge, the emission is purely dominated by the contribution of the disc of the galaxy. The far end of Fig. 3 represents the emission of the contribution of the arms of the galaxy. The maximum surface brightness at the center of the nucleus $\left(m_{B} / \operatorname{arcsec}^{2}=\right.$ 20.2, $\mathrm{m}_{\mathrm{V}} / \operatorname{arcsec}^{2}=19.5$ and $\left.\mathrm{m}_{\mathrm{R}} / \operatorname{arcsec}^{2}=18.7\right)$. The eastern part of the galaxy is brighter than the western part by $\left(0.94 \mathrm{mag} / \mathrm{arcsec}^{2}\right)$ in the $\mathrm{V}$ band $\left(21.07 \mathrm{mag} / \operatorname{arcsec}^{2}\right)$ as opposed to $\left(22.01 \mathrm{mag} / \operatorname{arcsec}^{2}\right)$. 


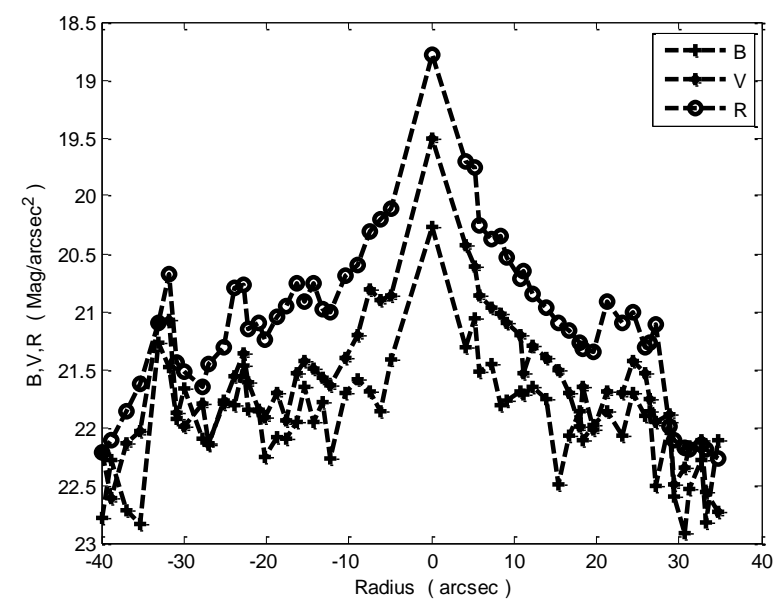

Fig.3: Photometric profiles along the major axis of NGC 4448.

Figs.(4a) and (4b) show the unusual dependence for the ellipticity and position angle of the isophotes on distance from the center. The circumnuclear region has nearly circular isophotes $(e \simeq 0.05)$ oriented in position angle $95^{\circ}$ Further variations in

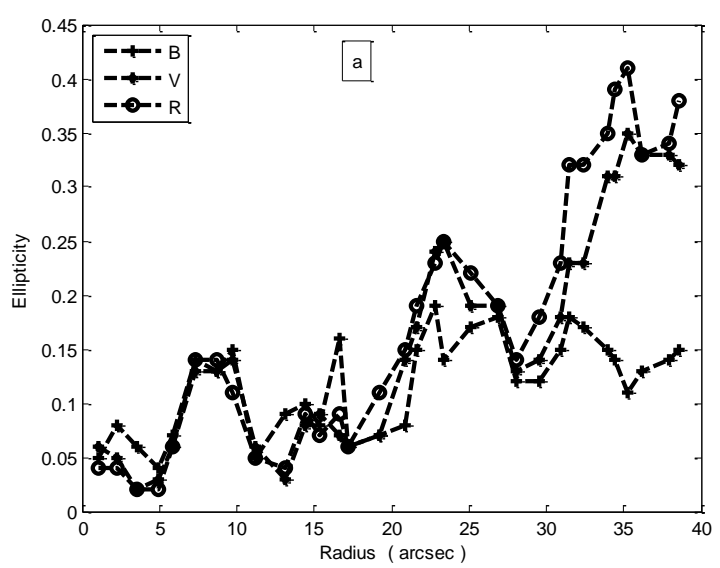

the position angle and, in part, the ellipticity of the isophotes are associated with the fact that the center of the bright ring does not coincide with the center of the galaxy's nucleus at distances $r>30^{\prime \prime}$ from the center.

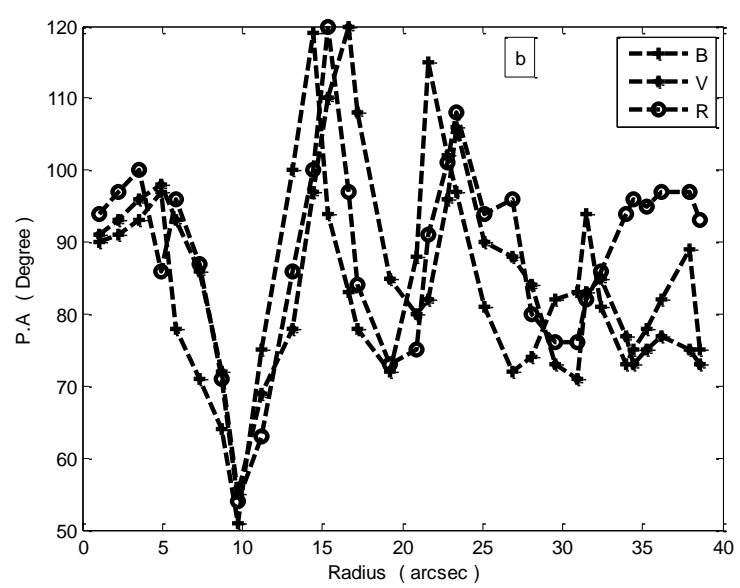

Fig. 4: (a) Isophote ellipticity (b) position angle of the galaxy $P$. A as a function of the distance $r$ to the center of NGC 4448.

Fig. 5 shows the $(\mathrm{B}-\mathrm{V})$ and $(\mathrm{V}-\mathrm{R})$ color indices along the major axis of NGC 4448. The galaxy becomes bluer with distance from the center, most strongly in $(\mathrm{B}-\mathrm{V})$. (B-V) varies from 1.35 mag in the nucleus to 0.58 mag at the periphery of the galaxy; the corresponding indices for $(\mathrm{V}-\mathrm{R})$ are 0.85 mag and 0.35 mag respectively. The color indices in the bar for $(\mathrm{B}-\mathrm{V})$ is $1.05 \mathrm{mag}$, and for $(\mathrm{V}-\mathrm{R})$ is $0.77 \mathrm{mag}$. 


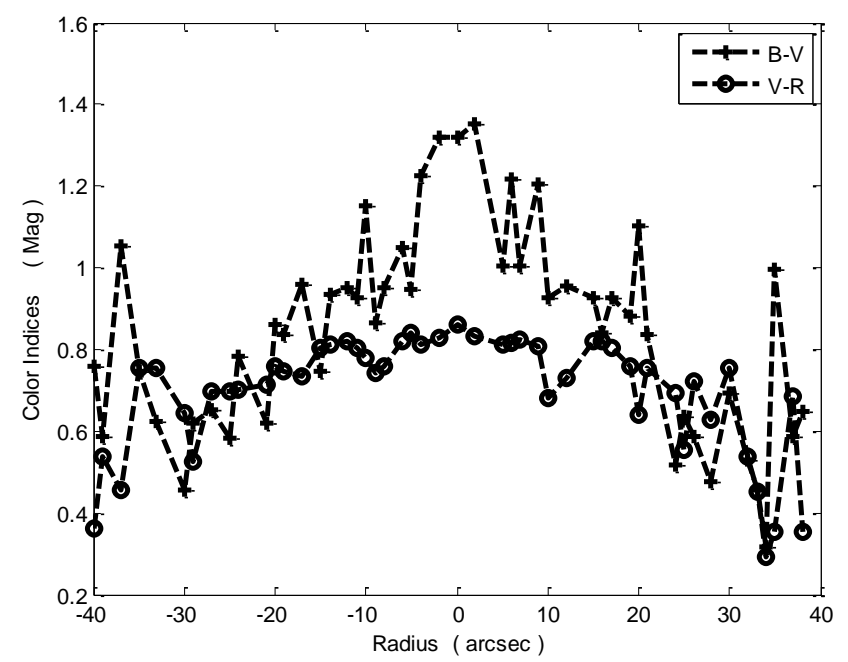

Fig.5: Profiles of the color indices along the major axis of the galaxy NGC 4448.

\section{Conclusions}

The eastern part of the galaxy NGC 4448 is brighter than the western part. This means that the brighter part have many constellations of young stars than that of lower brightness. NGC 4448 is one of the bluest galaxies in its class, and star formation has actively occurred. The star formation rate in the nucleus and spiral arms has remained virtually constant in time (as we can conclude from their positions in twocolor diagrams). This means that either the efficiency of star formation has increased in time in these regions or there is a constant input of gas to the nucleus and spiral arms of the galaxy.

\section{References:}

[1] J. Schombert and A.K. Smith, 2011. Systematic Bias in 2MASS Galaxy Photometry. astroph.IM.arXiv: 1107.1728v1

[2] P.A.Martinez. "A Practical Guide to CCD Astronomy". Cambridge University, 1998.

[3] R.Wodaski. Ron. "The New CCD Astronomy" New Astronomy Press., 2002.
[4] Zeilik, Michael and Stephen Gregory. "Astronomy and Astrophysics". $4^{\text {th }}$ ed. Saunders College Publishing, 1998.

[5] S. B. Howell, "Handbook of CCD Astronomy". Cambridge: Cambridge University Press. 2000.

[6] W. J. Kaufman and G. A. Freedman, "Universe" ( $5^{\text {th }}$ Ed.). New York: W. H. Freeman and Company, 1998.

[7] S.H. Ali and S.A. Albakri, Iraqi Journal of Physics, 12, 24 (2014) 8186.

[8] A. Halton, "Atlas of Peculiar Galaxies". Astronomy and Astrophysics Journal, 14 (1966) 1A.

[9] H.M. Hernandez, J.D. Zendejas, R.V. Avila, The Astronomical Journal, 134 (2007) 2286-2307.

[10] A.S. Gusev and S.S. Kaisin, Astronomy Reports, 46, 9 (2002) 712720.

[11] S.H. Ali, Iraqi Journal of Science, 56, 3A (2015) 2096-2103.

[12] Landolt, Astronomy Journal, 104, 340 (1992). 